\title{
Examination of Annular-Electrode Spark Discharges in Flowing Oxygen - Experimental Nuances
}

\author{
Darren C. Tinker ${ }^{1}$, Marsalis Pullen ${ }^{2}$ \\ Vanderbilt University, Nashville, TN, 37212 \\ And \\ Robin J. Osborne ${ }^{3}$ \\ ERC, Inc., Jacobs Space Exploration Group, Huntsville, AL 35812 \\ And \\ Robert W. Pitz \\ Vanderbilt University, Nashville, TN, 37235
}

\begin{abstract}
Microsecond sparks and the resulting plume of hot gas/plasma were examined against a parametric pressure-distance matrix. Schlieren imaging is used to capture the spatial and temporal location of spark discharge exhaust for two milliseconds. Low pressure and larger gap widths created the largest size and intensity signal for the spark-affected plumes. Experimental exit-plume velocities trend well with analytic predictions using a mean pressure between the chamber and atmospheric conditions. Due to the quadratic relation of the annulus area and gap width, larger gap width velocities are more accurately represented by analytic predictions using atmospheric pressure as the larger exit area restricts the flow less. The same pressure adjustment, when applied to breakdown voltages, improves data alignment with Paschen's Curve.
\end{abstract}

\section{Introduction}

Electrical discharges are commonplace in the realm of ignition and are followed by gaseous kernels that act as the catalyst for combustion reactions. Present literature focusing on plasma assisted combustion (PAC) or plasma assisted ignition (PAI) primarily refers to non-thermal plasmas discharging on nanosecond timescales with equilibrium plasmas receiving less attention [1]. More traditional sparks last microseconds and consist of a hot core with cooled fringes surrounding the arc [2]. They create thermal (equilibrium) plasmas and impart considerable energy to the fluid where the arc flashes. While microsecond sparks require more energy than their nanosecond counterparts, the working fluid not only gains dissociated species, but also a temperature rise to help initiate combustion.

For spark ignition of combustion devices, studies have ranged from investigating the electrical characteristics $[3,4,5]$ to imaging of ignition kernels post-discharge [6, 7]. Ample literature involves pronged electrodes with air-hydrocarbon mixtures, which had electrical input parameters and geometric shapes (e.g. cones, prongs) comprehensively varied $[6,8,9]$. Often driven by specific applications, such work revolves around stagnant conditions, which limits studies of sparks in flowing gases. Sforzo et al.

${ }^{1}$ Graduate Student, VU Station B \#351592, 2301 Vanderbilt Place. Student Member.

${ }^{2}$ Graduate Student, VU Station B \#351592, 2301 Vanderbilt Place. Student Member.

${ }^{3}$ Senior Engineer, Mail Stop ER36, NASA Marshall Space Flight Center. Associate Fellow.

${ }^{4}$ Professor \& Department Chair, VU Station B \#351592, 2301 Vanderbilt Place. Fellow. 
characterized the kernel formation for short duration discharges $(<1 \mu \mathrm{s})$ both numerically and experimentally. However, the study is restricted to pronged electrodes at a fixed length and atmospheric pressure [10]. In flowing propane-air, Ballal and Le Lefebvre showed that turbulence has a minimal influence on the energy deposited by the spark and that spark durations decreased with higher velocities, yet involved reacting flow [3].

The aim of this paper is to shrink the gap of knowledge for sparks in flowing oxygen through an annular electrode pair. This paper is an investigation of the quenching effects, analytic validation, and optical measurements of spark discharges in flowing oxygen; and an extension of the analysis of Tinker, Osborne, and Pitz [11].

\section{Experimental Setup}

Measurements of the exhaust plumes were taken with Schlieren imaging. A schematic of the original system is shown in prior work [11] with the current system using a different light source and additional pressure measurement. A schematic of the current system sans schlieren equipment is shown in Figure 1. The spark gap widths and pressures used for the test matrix are shown in Table 1. For each pressure setting, the supply pressure (pressure 1) remained constant with a maximum range of 5 psia. The downstream venturi (pressure 2) and pre-gap chamber (pressure 3) pressures had greater variance due to the change in exit area. The exit annulus area was quadratically related to the spark gap width. The area to gap width ratios are listed for reference.

Table 1 - Spark gap electrode diameters, and pressures used in experiment. $\mathrm{P}_{1}$ is the Supply Pressure. $\mathrm{P}_{2}$ is the pressure downstream of the choke venturi. $\mathrm{P}_{3}$ is the pressure immediately before the spark gap chamber

\begin{tabular}{c|c|c|c|c|c}
$\begin{array}{c}\text { Spark } \\
\text { Gap }\end{array}$ & $\begin{array}{c}\text { Outer Dia. } \\
{[\mathbf{m m}]}\end{array}$ & $\begin{array}{c}\text { Inner Dia. } \\
{[\mathbf{m m}]}\end{array}$ & $\begin{array}{c}\text { Width } \\
{[\mathbf{m m}]}\end{array}$ & $\begin{array}{c}\text { Area } \\
{\left[\mathbf{m m}^{2}\right]}\end{array}$ & $\begin{array}{c}\text { Width } \\
\text { to Area }\end{array}$ \\
\hline SG 1 & 3.43 & 2.39 & 0.51 & 4.77 & 2.8 \\
\hline SG 2 & 4.00 & 2.39 & 0.79 & 8.00 & 2.5 \\
\hline SG 3 & 4.50 & 2.39 & 1.04 & 11.4 & 2.3 \\
\hline SG 4 & 4.98 & 2.39 & 1.30 & 15.0 & 2.2 \\
\hline N/A & - & - & - & - & - \\
\hline SG 6 & 6.02 & 2.39 & 1.80 & 24.0 & 1.9 \\
\hline N/A & - & - & - & - & - \\
\hline SG 8 & 7.04 & 2.39 & 2.31 & 34.4 & 1.7
\end{tabular}

\begin{tabular}{c|c|c|c}
$\begin{array}{c}\text { Pressure } \\
\text { Setting }\end{array}$ & $\begin{array}{c}\text { Nominal } \\
\mathbf{P}_{1}[\mathbf{p s i a}]\end{array}$ & $\begin{array}{c}\text { Nominal } \\
\mathbf{P}_{2}[\mathbf{p s i a}]\end{array}$ & $\begin{array}{c}\text { Nominal } \\
\mathbf{P}_{3}[\mathbf{p s i a}]\end{array}$ \\
\hline PS 1 & 35 & 19 & 17 \\
\hline PS 2 & 55 & 22 & 20 \\
\hline PS 3 & 75 & 26 & 23 \\
\hline PS 4 & 95 & 31 & 28 \\
\hline PS 5 & 115 & 37 & 33 \\
\hline PS 6 & 140 & 44 & 40 \\
\hline PS 7 & 165 & 52 & 47 \\
\hline PS 8 & 190 & 59 & 54 \\
\hline PS 9 & 215 & 67 & 61 \\
\hline PS 10 & 264 & 82 & 74 \\
\hline PS 11 & 315 & 97 & 88 \\
\hline PS 12 & 363 & 112 & 102
\end{tabular}

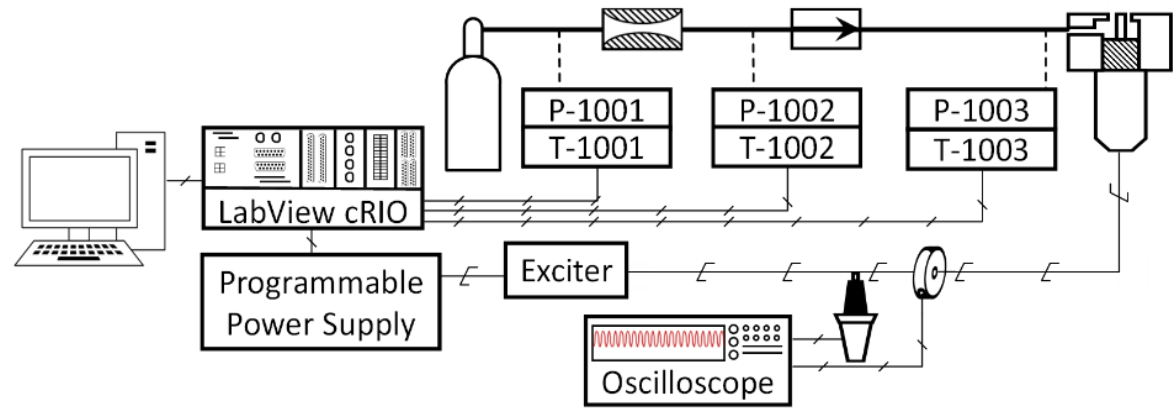

\begin{tabular}{|l|}
\hline \multicolumn{1}{|c|}{ Key } \\
Current Monitor \\
Check Valve \\
Choke Venturi \\
High Voltage Probe \\
Pressure Transducer \\
Spark Igniter \\
Thermocouple
\end{tabular}

Figure 1 - Instrumentation schematic and measurement locations 
The optical setup contained two f/10 $203 \mathrm{~m}$ diameter parabolic mirrors spaced $2 \mathrm{~m}$ apart. The knife edge and light source slit were set $180 \mathrm{~mm}$ from the collimated light centerline. The light source was a UHP High Intensity Light Source ILP-2, which passed through a liquid light-guide cable and was expanded to a 15 degree beam angle $\left(\theta_{1}\right)$ using a Lumatec two optic quartz lens. The light was passed through a condenser array with two $50 \mathrm{~mm}$ diameter, $\mathrm{f} / 2\left(\mathrm{f}_{13}, \theta_{3}\right)$ achromats as shown in Figure 2. The second achromat focused on a horizontal slit, and the beam exiting was cropped by an iris aperture (not shown). The mirror captured $68 \%$ of the incoming light $\left(\mathrm{d}_{3}\right)$, which was sufficient to resolve an image with one microsecond exposure time on a Phantom v310 high speed camera with a Sigma aspherical 170-500 mm f/5-f/6.3 lens. For this experiment, an exposure time and extended dynamic range of $9.3 \mu \mathrm{s}$ were used to provide ample contrast for post-processing. Each video contained 100,000 frames with a capture rate of 100,000 FPS. For post-processing, two milliseconds (200 frames) were extracted following an image based trigger. Spatial resolution was $200 \mu \mathrm{m}$, as determined by a Ronchi grating, and the total image size was 160 x 127 pixels at 4.1 pixels per $\mathrm{mm}$.

A detailed explanation of the high-speed video post-processing procedure is found in the preceding study [11]. In short, an image based trigger determined time zero for each spark. A spatial average of signal was taken across all events with the same timestamp. Spatially averaged images were concatenated into a single video. The weighted centroid was calculated based on signal intensity, and a standard centroid was calculated after a secondary binary processing. Differences in weighted centroid locations versus timestamps were used to calculate plume velocity. Integrating the spatially averaged video over time resulted in a single image, where intensity correlated to the absolute magnitude of deflection from the schlieren images.

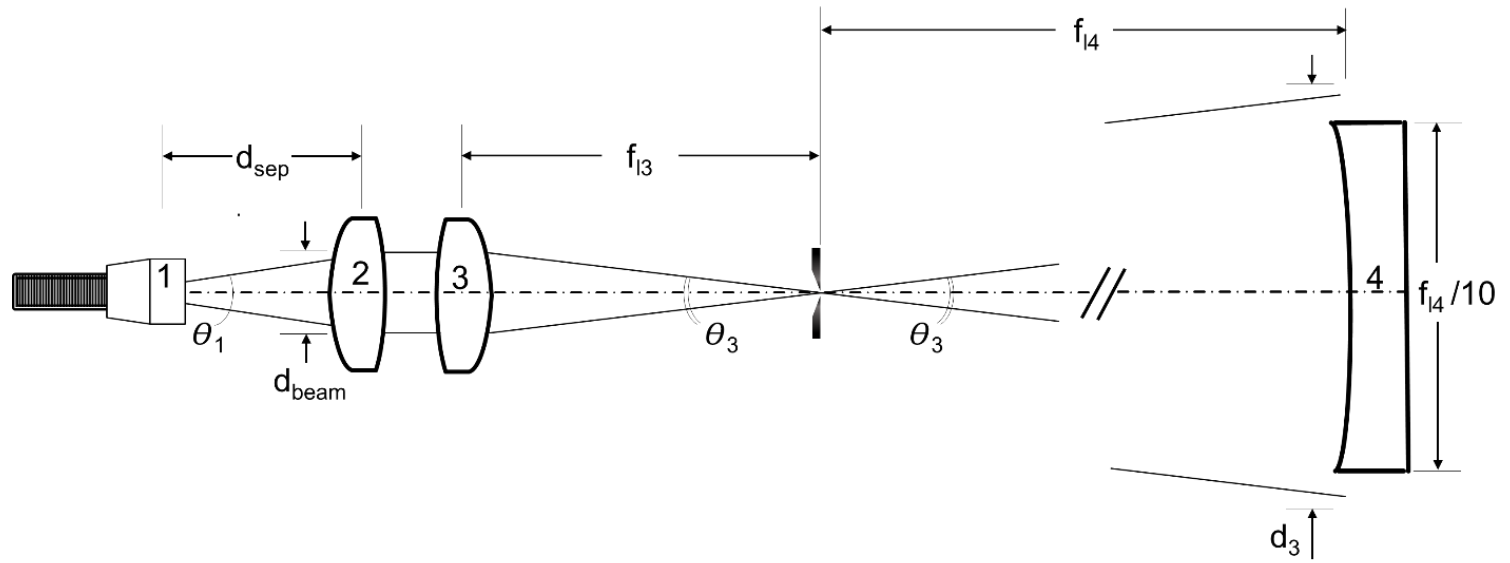

Figure 2 - Light source and condenser array schematic for Schlieren setup

Electrical data was measured by a model 410 Pearson current monitor $(50 \Omega$ termination, thus lowering sensitivity by a factor of two), model P6015 Tektronix high voltage probe (1M $\Omega$ termination), and recorded by a Teledyne LeCroy WaveSurfer 44MXs-b oscilloscope. The trigger channel was on the high voltage probe with an $800 \mathrm{~V}$ threshold. Spatial resolution was $2.5 \mathrm{kV}$ per division, and 1 A per division with offsets of negative $7.5 \mathrm{kV}$ and negative $3 \mathrm{~A}$ respectively. Temporal resolution was $1 \mathrm{~ns}$ over a $100 \mu$ s duration for 249 triggered events. Each data point consists of four trials with flowing gas and spark discharges - four videos and four electrical datasets - and one trial with flowing gas and no spark discharges - one background video. Instantaneous power was integrated to determine mean energy for each data point. The RSMD of mean energy was used to determine the spark duration. A central difference with 30 points was used to ensure robustness and repeatability in calculations. The results are shown in Figure 3 with the mean duration lasting $44 \mu \mathrm{s}$. 


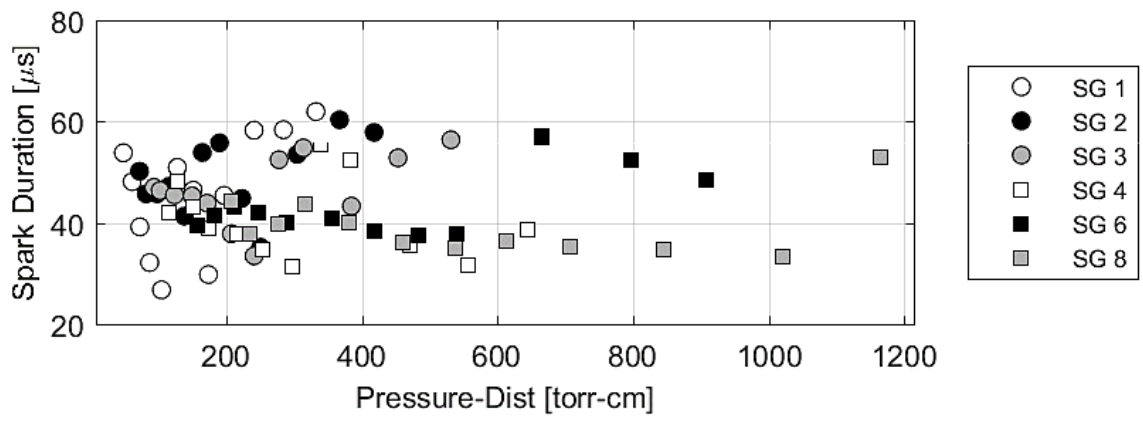

Figure 3 - Spark duration versus the pressure distance product shown for all spark gaps

Mass flow was regulated by a Fox choke venturi with a $1.3 \mathrm{~mm}$ diameter throat. Choked conditions were confirmed for each test as shown in
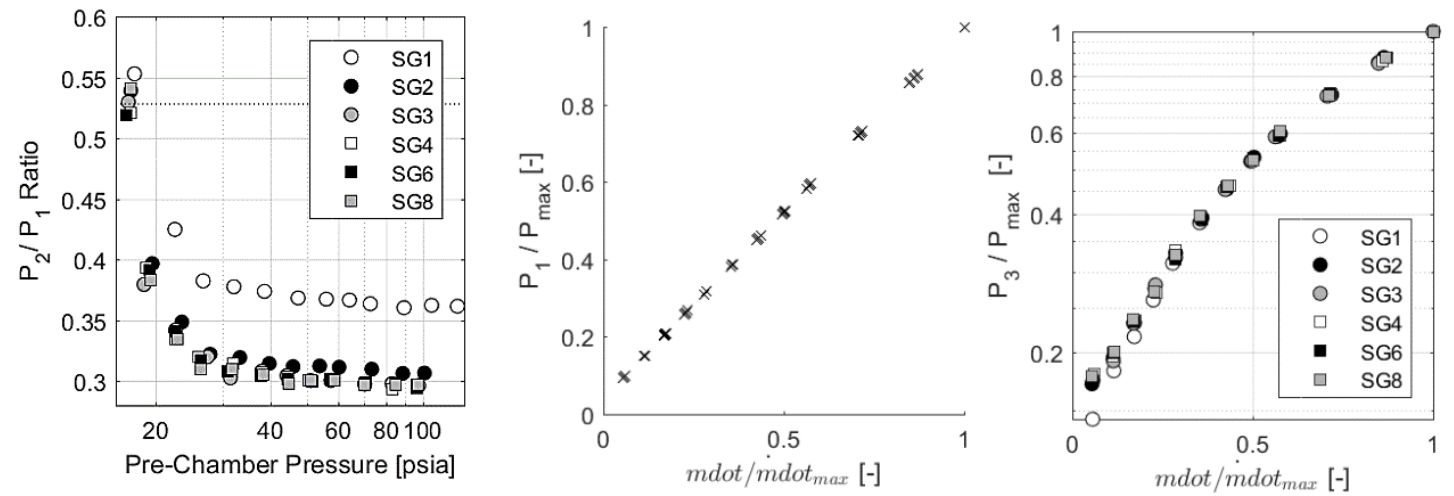

Figure 4, which includes mass flow rates and pressures for each test. Fluid properties were measured using Omega Type K open tip, ungrounded, $0.025 \mathrm{~mm}$ diameter wire thermocouples, and three Omega PX309$500 \mathrm{~A} 5 \mathrm{~V}$ pressure transducers. Two transducers were upstream $\left(\mathrm{P}_{1}\right)$ and downstream $\left(\mathrm{P}_{2}\right)$ of the venturi as in the previous study, and a third was added directly before the spark gap chamber $\left(\mathrm{P}_{3}\right)$. Data was recorded by a LabView cRIO-9064. Sparks were generated using a Champion Aerospace CH31887-3 spark igniter connected to a capacitive Champion Aerospace SK927123 exciter, which was powered by a Tektronix PWS4305 DC power supply. Pure oxygen was used as the working fluid. The oxidizer was injected into a cavity upstream of the spark gap, then directed through an annulus formed by two electrodes as shown in Figure 5. The outer electrode was made of stainless steel, while the inner electrode was made of a Nickel alloy. The chamber inlet was widened to the nominal tube diameter. This was to prevent a secondary choke point found in previous work [11], and to provide a more accurate measurement of the chamber pressure. Sparks formed within the annulus and were extended outward by the flow. The excited gas was carried downstream by the bulk flow where it inevitably reached equilibrium.
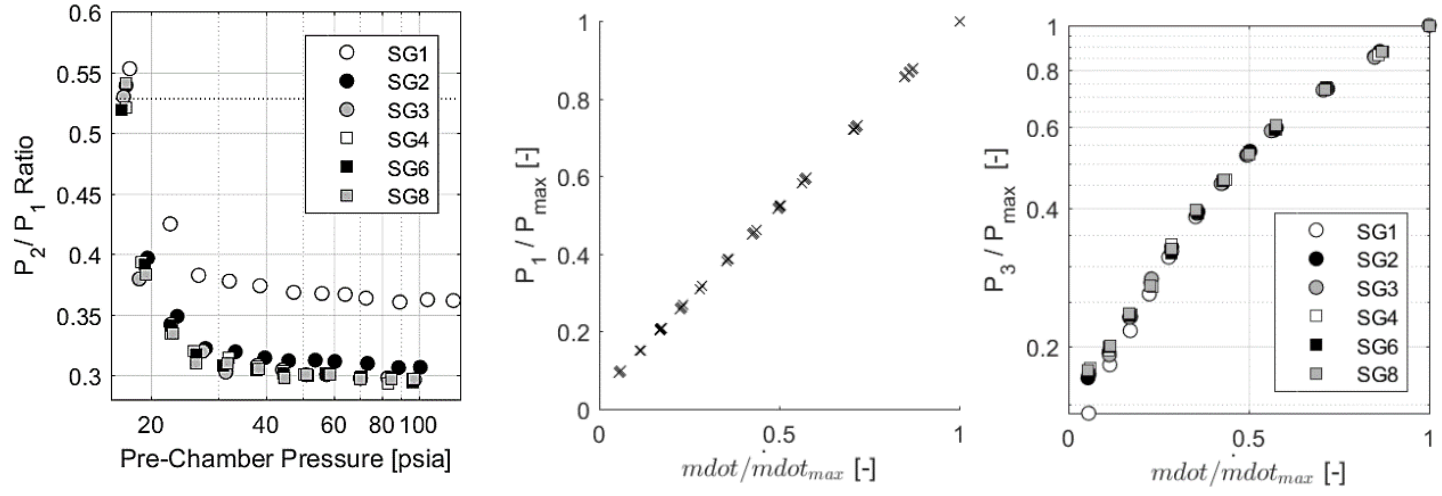
Figure 4 - Pressure ratios across choke venturi $\left(\mathrm{P}_{1} / \mathrm{P}_{2}\right)$, normalized supply pressure $\left(\mathrm{P}_{1}\right)$ versus normalized mass flow rate, and normalized pre-gap chamber pressure $\left(\mathrm{P}_{3}\right)$ versus normalized mass flow rate

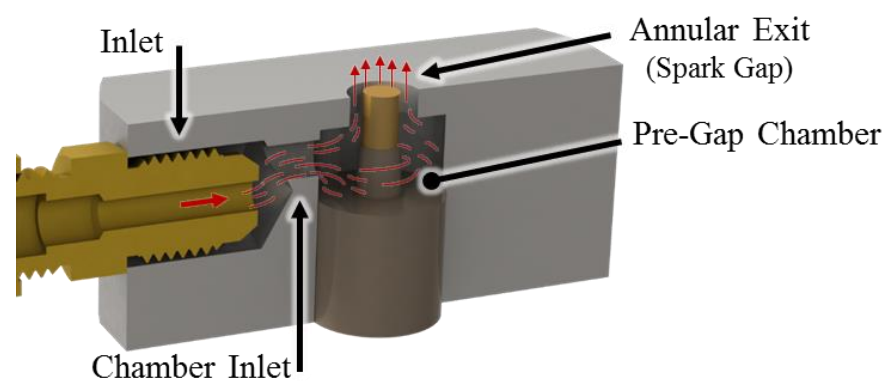

Figure 5 - Spark gap configuration. Axial symmetry is only for the annulus and not the chamber body

\section{Results and Discussion}

Quenching occurs when there is insufficient energy to ionize the medium within the spark gap. Since there is no electron avalanche and thus no conductive plasma between the electrodes, there is no current flow. The potential energy created from the exciter is dissipated without a discharge, and electrically rings within the high voltage leads between the spark exciter and spark igniter. As this study requires nonintrusive electrical measurements to the flow path, the probes are located midway through the high voltage leads. In turn, the ringing caused by quenched sparks has significant effects on electrical measurements, and can easily be overlooked when dealing with automated processing of large datasets.

For results in this paper, when spark quenching was detected, the associated electrical data was discarded as it had no physical relevance to the study at hand. For each data point, 996 events were triggered from high voltage spikes caused by quenching or breakdown. Across all spark gap widths and pressures, 95,616 events were recorded. Of this total, only $6.6 \%$ events measured were from spark quenching. The largest number of quenched events reached 949 or $95 \%$ for PS12, SG2. A surface map across all conditions is shown in Figure 6 with quenching calculated as a percentage of the total number of events per data point.

After the data for quenched conditions were removed from the dataset, the values for breakdown voltage were examined. The surface map of breakdown voltage versus the pressure-distance product steadily rises from the bottom left to top right as shown in Figure 7, which is expected from theory. A comparison of the experimental results to the theoretical prediction by Paschen's law is shown in Figure 8. Results were in closer agreement to Paschen's curve than previous efforts [11] by over an order of magnitude. This is attributed to widening the chamber inlet (see Figure 5 for reference) to roughly the size of the tube diameter to prevent choked flow and a major pressure loss after the pressure measurement. Some deviations are observed, yet follow suit with work from other groups [3] and are accepted because of nonuniformity of the electric field. For higher pressure-distance measurements, it is hypothesized that the pressure at the gap is lower than the pressure measurement immediately preceding the chamber, and likely lies closer to atmospheric conditions. This hypothesis is discussed and supported subsequently.

Removal of quenched data had a large impact on the mean energy measurements. By excluding quenched signals, the mean energy dropped as much as $21 \%$ for select cases. The surface map for mean energy is substantially smoother, shown in Figure 9 with the only major drop in the region with low pressures and small spark gaps. The current measurements for these cases were saturated, and the differences are attributed to experimental limitations instead of a physical phenomenon. 
AIAA Science and Technology Forum (AIAA SciTech 2019)

Grand Hyatt, San Diego, CA, 7 - 11 Jan 2019

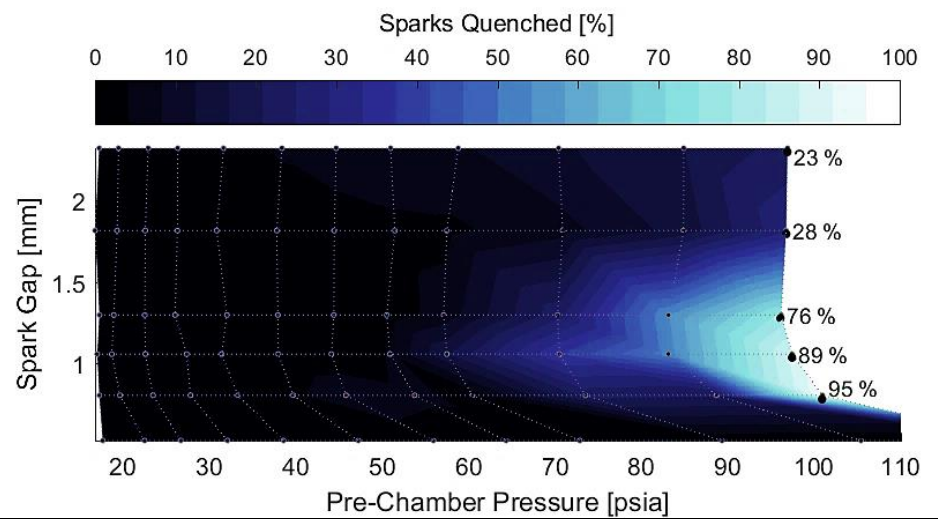

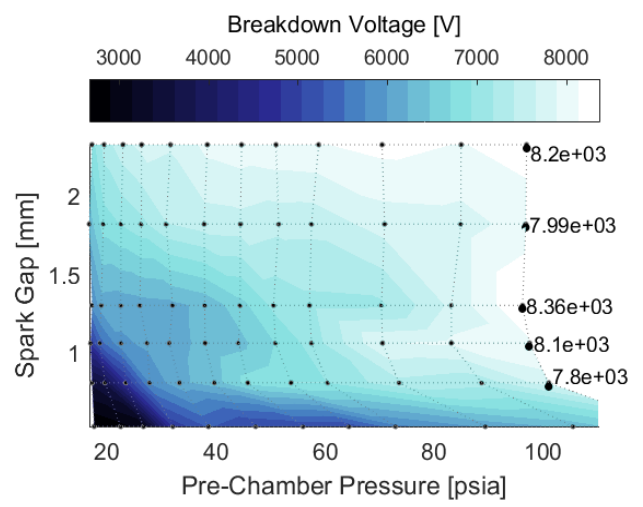

Figure 7 - Surface map of experimental breakdown voltages per spark gap and pressure
Figure 6 - Spark events quenched per data point across 996 triggered events

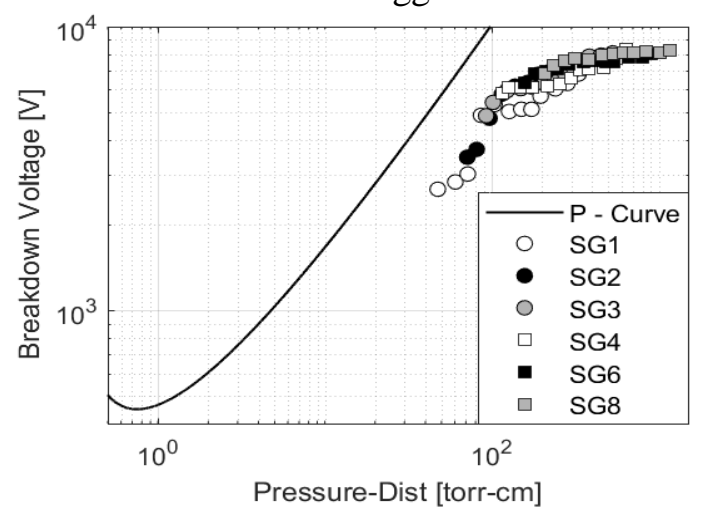

Figure 8 - Experimental breakdown voltages compared to Paschen's curve 


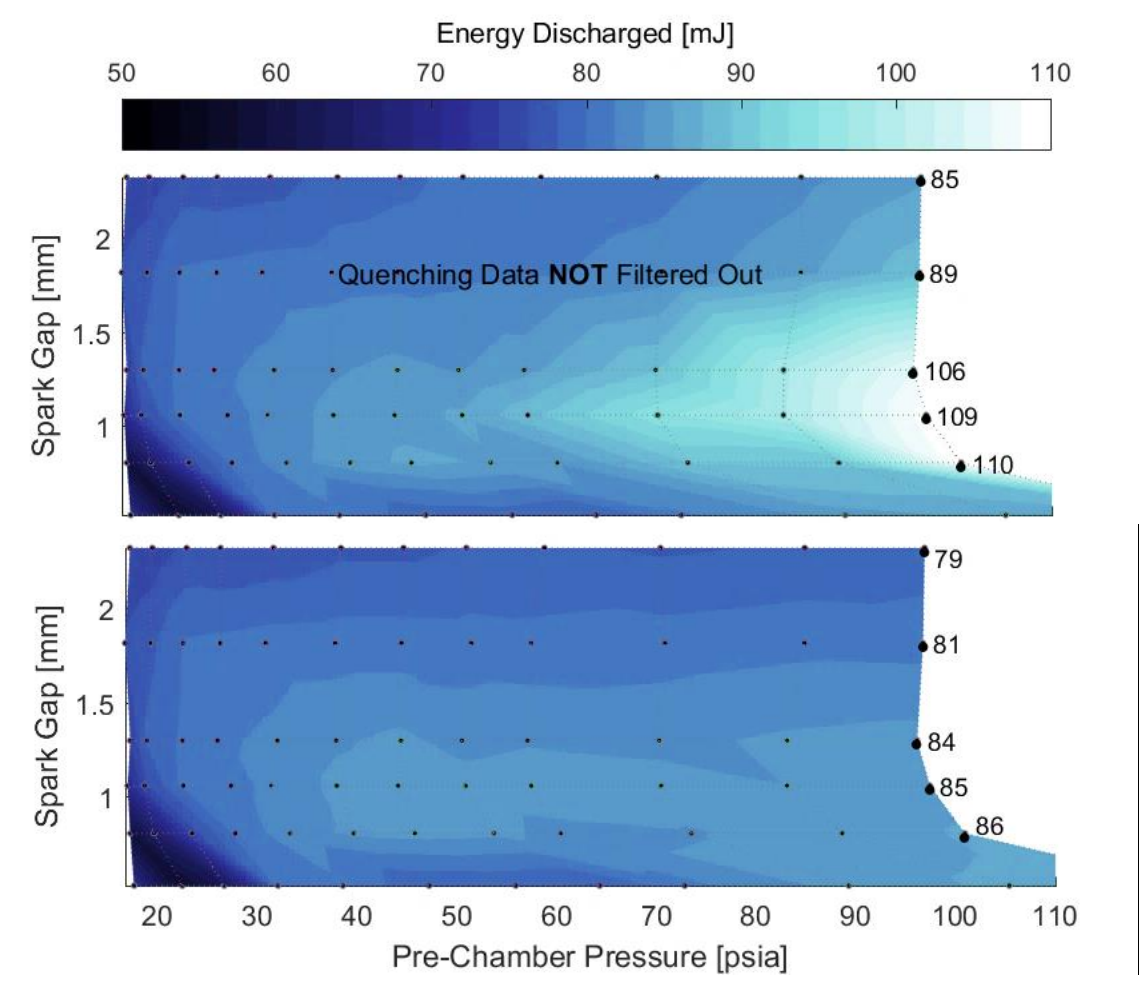

Figure 9 - Surface maps for spark mean energies with quenching included (top) and filtered out (bottom), where brightness corresponds to the signal magnitude

The Schlieren images were multipurpose, and were first used to calculate flow velocities at the annulus exit using a simple conservation of mass. The mass flow rate was from the choke venturi calculation; the exit area was the area between the electrodes; and the density was calculated as a function of pressure. The standard pressure used was the mean value between pre-chamber pressure $\left(\mathrm{P}_{3}\right)$ and atmospheric pressure.

A spatial sum of the exhaust was calculated from the entire set of images to determine the optimal spark gap width and pressure combination. Optimal in this case implies that a large volume of gas over a small time, or a small volume of gas over a large time frame would be equal in value as the effective volume heating would be similar. Figure 10 shows sample frames of the exhaust cloud being carried and turbulently dissipated by the surrounding fluid. The cloud is a mean value per frame across all sparks captured. Largely all clouds were observed with a cylindrical starting shape that was dissipated from the higher velocity gases behind it. Experimental studies with larger spark gaps, stronger and faster sparks, and accompanying simulation results [10] have shown similar behaviors, and discuss the shape transforming to a toroidal form.
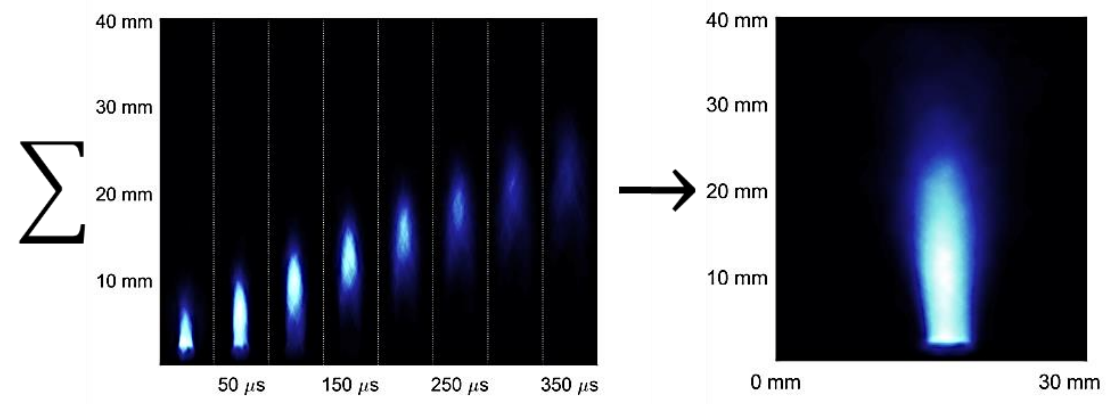

Figure 10 - Sample frames of an exhaust cloud, and pictorial depiction of a temporal sum result

The optical flow velocity trended well with analytic predictions using a simple mass flow equation. As mentioned, mass flow was calculated using the supply pressure and fluid temperatures upstream of the 
venturi. Area was determined using the electrode diameters. Density was calculated as a function of pressure, where three distinct pressure levels were used - pressure at infinity $\left(\mathrm{P}_{\infty}\right)$, pre-chamber pressure $\left(P_{3}\right)$, and a weighted mean pressure $\left(P_{\text {wt.mean }}\right)$ between infinite and measured pressures.

The mean value was calculated using Equation 1,

$$
P_{w t . m e a n}=\frac{\left.\left(P_{3}+n \cdot P_{\infty}\right),\right)}{n+1}
$$

Where $\mathrm{n}$ is the weighting factor for pressure at infinity. For the majority of datasets, a 1:1 ratio (standard mean) of atmospheric pressure to pre-chamber pressure trended closely with experimental results.

However, for larger exit areas, using a pressure ratio of 4:1 $\left(\mathrm{P}_{\infty}: P_{3}\right)$ for the weighted mean shifts results substantially closer to experimental velocities. The quadratic relation between the exit area and spark gap width is the explanation for the variance. As the gap width increases, the area increases by a square power. Thus, larger spark gaps have substantially less flow resistance and the pressure within the gap may be substantially lower than the pre-chamber measurement at $\mathrm{P}_{3}$. For sufficiently large areas, analytic velocities could be accurately calculated using only the atmospheric pressure. Succinctly, calculating the spark-gap fluid density using $\mathrm{P}_{\infty}$ is the upper bound of velocity exiting the gap, and $P_{3}$ is the lower bound. Results comparing of the experimental flow velocity and all three analytic methods are shown in Figure 11.

It can be inferred that the pressure measured prior to the spark chamber does not match the pressure at the spark gap for moderate to large spark gaps in this study, which would shift the results of breakdown voltage (see Figure 8 for reference) closer to the theoretical values in Paschen's curve. For completeness, Figure 12 shows the results if a 1:1 ratio (applicable to moderate gaps) of atmospheric and measured pressure are used as well as a 4:1 ratio (applicable to large gaps). As a secondary property matches closer to theory by this assumption, the implication is that weighting the pressure used to calculate an analytic velocity is reasonable.

For the smallest exit area - smallest gap width - the pressure at the gap is closer to $\mathrm{P}_{3}$. Velocities calculated from pre-chamber pressures match well for low to moderate flow rates. Near 60 psia, the exit velocity peaks and remains elevated compared to the preceding trend. For the high flow rates, the mean pressure velocity prediction matches experimental results well. It is hypothesized that an asymmetric vena contracta is formed at the corner of the outer electrode (depicted in Figure 11). The corner boundary layer narrows the flow channel, creating an effective nozzle and accelerating the flow to sonic conditions at the throat (vena contracta diameter). In turn, the flow within the spark gap can become supersonic. This assumption would explain the pressure drop and external velocity increase. 

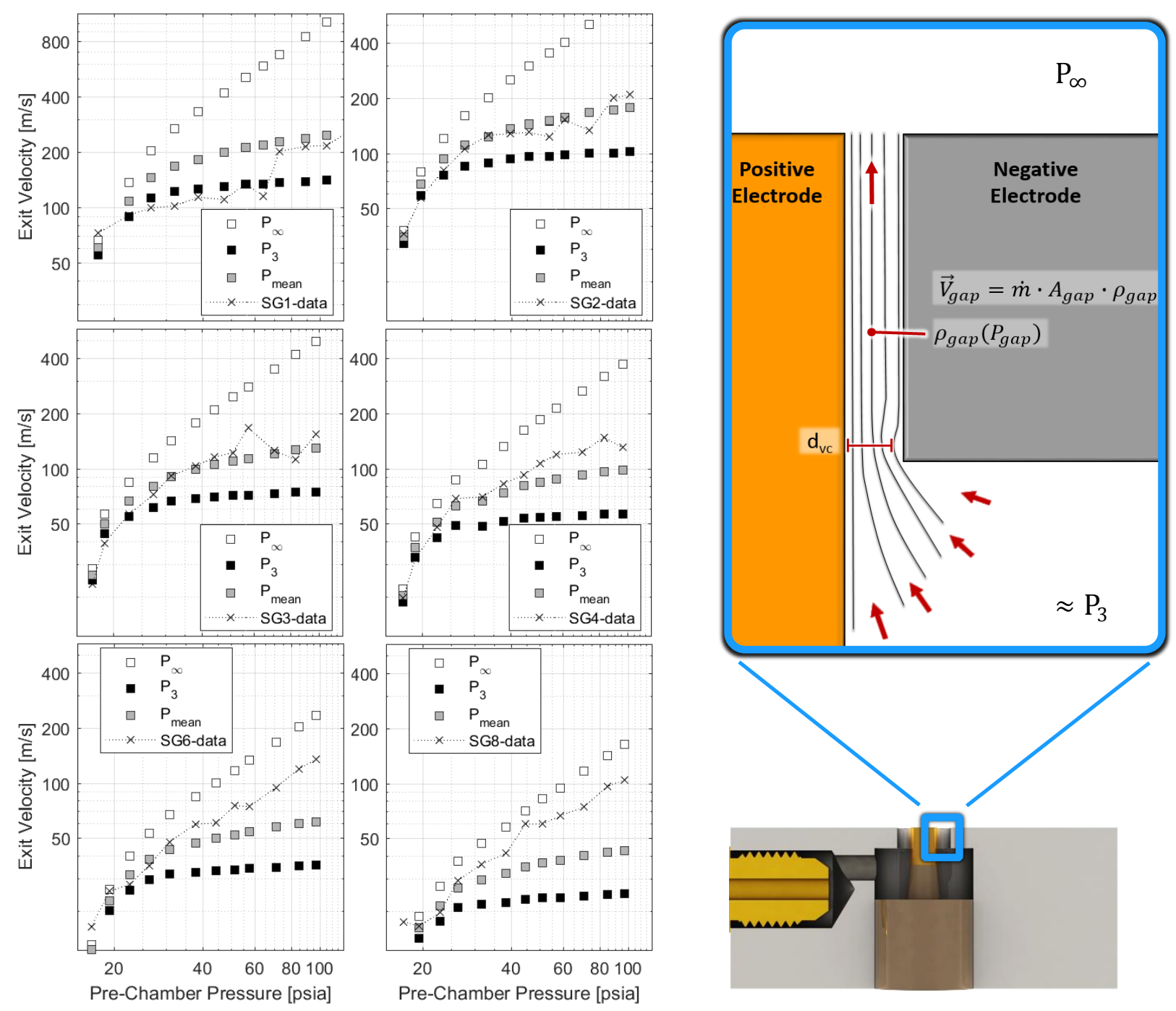

Figure 11 - Experimental velocity calculations and analytic predictions using three different pressures for the density calculation at the spark gap
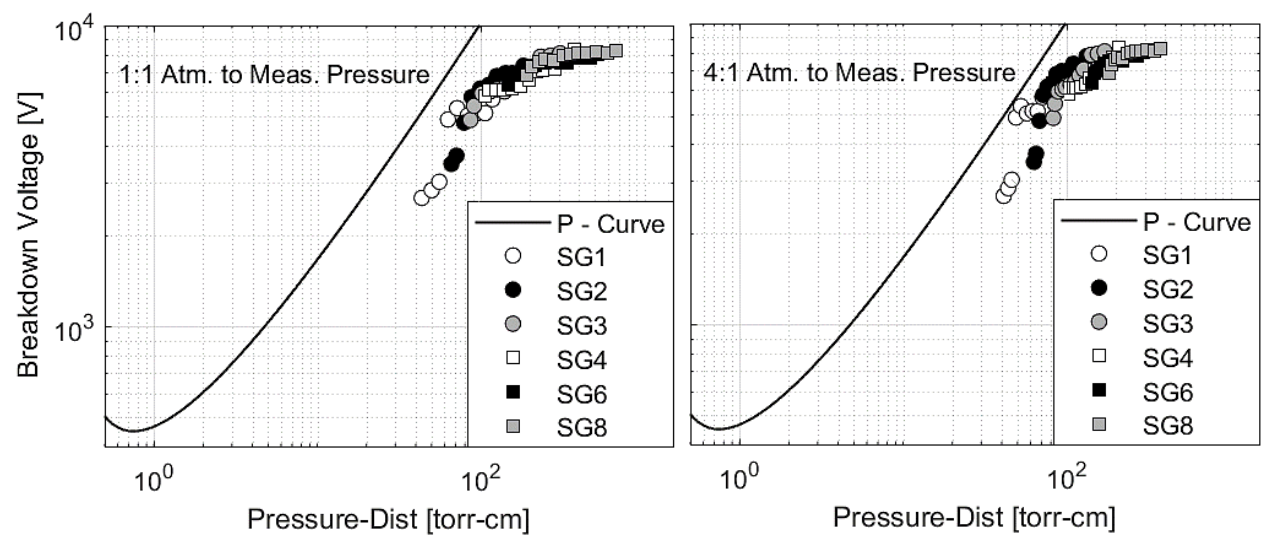

Figure 12 - Comparisons of Paschen's curve and experimental breakdown voltages vs. pressure-distance products where the pressure is weighted toward atmospheric conditions 
The plasma cloud/hot exhaust following the spark discharge is the last piece examined here. As shown in Figure 13, a temporal sum was calculated for each spark gap width and pressure setting. The resulting image shows a weighted average of where the hot exhaust cloud will be, and would likely impinge on a combustible mixture in application. The full matrix of temporal sums is shown in Figure 13, but with the caveat that quenching is not accounted for. As the processing method relied on an image based trigger, results are not normalized for the total number of sparks that actually occurred. This is clearly seen for spark gap two and pressure twelve, where the result looks equivalent to spark gap three and pressure four where virtually no quenching occurred.

To account for quenching and provide a more quantitative interpretation of this data, a second summation was performed across space reducing each image to a single point that corresponds to a space and time independent plume intensity. The absolute values were multiplied by a quenching factor (one minus percent quenched) and plotted as a surface map, which is shown in Figure 14. Here, it is clear that the highly quenched zones are the least favorable if aiming for an optimal plasma/exhaust cloud.

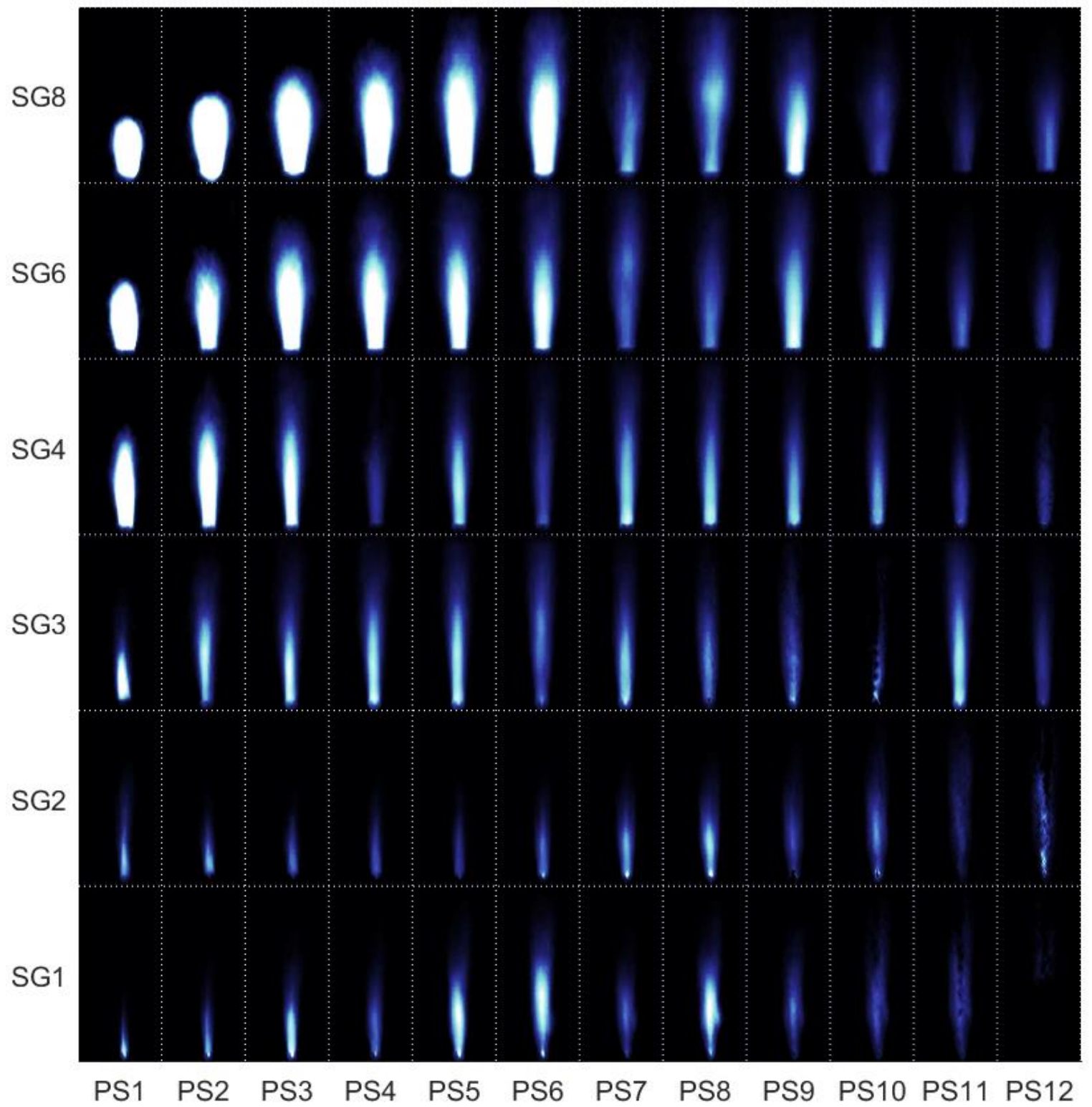

Figure 13 - Time integration of spatially averaged signals per spark gap and pressure combination (enhanced contrast). 


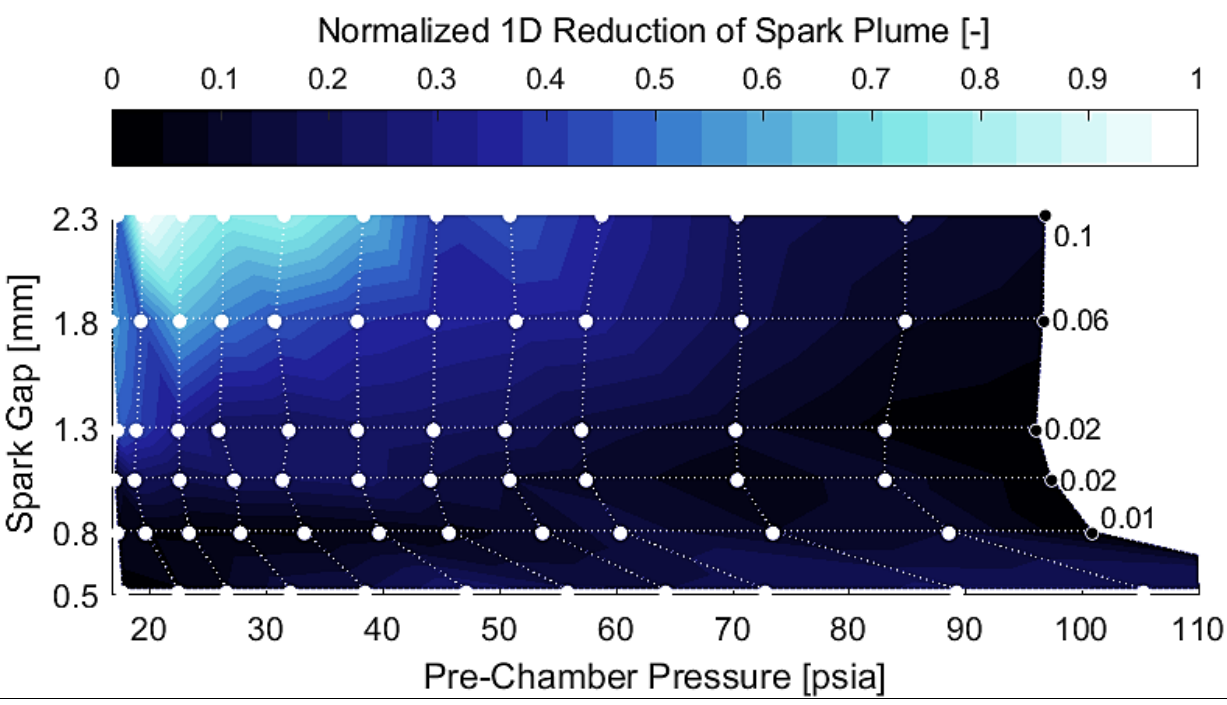

Figure 14 - One dimensional reduction of spark-affected plumes by a temporal and spatial integration. White circles correspond to individual data points, and correlate to time averaged images of Figure 13

\section{Conclusions}

Microsecond sparks and the resulting plume of hot gas/plasma were examined against a parametric pressure-distance matrix. Schlieren imaging is used to capture the spatial and temporal location of spark discharge exhaust for $2 \mathrm{~ms}$ total with a $10 \mu$ s resolution between frames. High voltage and current measurements are recorded at a $1 \mathrm{~ns}$ resolution for $100 \mu$ s for 996 triggered events. Effects of quenching are discussed, demonstrated, and accounted for when processing data.

The size of the spark exhaust plumes show that low pressure and large spark gap widths are optimal for creating the largest exhaust plumes. Reduction of the datasets to a single dimension by a temporal and spatial summation provides a succinct comparison for large data sets. Experimental research is necessary to determine what exhaust plumes are ideal for ignition.

Optically measured velocities are calculated using weighted centroids. These experimental values trend well with analytic predictions using a mean pressure between the chamber and atmospheric conditions. Due to the quadratic relation of the annulus area and spark gap distance, larger gap sizes (1.7:1, gap area to gap width) are more accurately represented by analytic predictions using atmospheric (or a weighted mean toward atmospheric) pressure as the flow is not strongly restricted at the exit.

With regards to a prior study [11], unaccounted quenching was shown to impact results when calculating breakdown voltage, mean energies, and spark duration in select cases. Furthermore, expanding the chamber inlet to prevent choked flow and a large pressure drop caused breakdown voltages to align closer with Paschen's curve.

\section{Acknowledgements}

This work is funded by the NASA Space Technology Research Fellowship for Darren C. Tinker. The authors acknowledge John Peugeot and Paul Dumbacher at NASA MSFC for their support of this project, as well as Tom Fleetwood at Champion Aerospace for the loan of the exciter. 


\section{References}

[1] Y. Ju and W. Sun, "Plasma assisted combustion: progress, challenges, and opportunities," Combustion and Flame, vol. 162, pp. 529-532, 2015.

[2] D. L. Flamm and O. Auciello, Plasma Diagnostics: Discharge Parameters and Chemistry, Volume 1, San Diego: Academic Press, 2013.

[3] D. R. Ballal and A. H. Lefebvre, "The influence of spark dischage characteristics on minimum ignition energy in flowing gases," Combustion and Flame, vol. 24, pp. 99-108, 1975.

[4] T. Yuasa, S. Kadota, M. Tsue, M. Kono, H. Nomura and Y. Ujiie, "Effects of energy deposition schedule on minimum ignition energy in spark ignition of methane/air mixtures," Proceedings of the Combustion Institute, vol. 29, pp. 743-750, 2002.

[5] J. Kleinhenz, C. Sarmiento and W. Marshall, "Spark ignition characteristics of a LO2/LCH4 engine at altitude conditions," in 48th AIAA/ASME/SAE/ASEE Joint Propulsion Conference, Atlanta, 2012.

[6] S. Bane, J. Ziegler and J. Shepherd, "Investigation of the effect of electrode geometry on spark ignition," Combustion and Flame, vol. 162, pp. 462-469, 2015.

[7] N. Kawahara, S. Hashimoto and E. Tomita, "Plasma temperature of spark discharge in a sparkignition engine using a time series of spectra measurements," in 18th International Symposium on the Application of Laser and Imaging Techniques to Fluid Mechanics, Lisbon, 2016.

[8] C. C. Swett, "Investigation of spark gaps subjected to altitude and air-velocity conditions," National Advisory Committee for Aeronautics, Washington, 1948.

[9] M. Kono, S. Kumagai and T. Sakai, "The optimum condition for ignition of gases by composite sparks," Symposium (International) on Combustion, vol. 16, no. 1, pp. 757-766, 1977.

[10] B. Sforzo, A. Lambert, J. Kim, J. Jagoda, S. Menon and J. Seitzman, "Post discharge evolution of a spark igniter kernal," Combustion and Flame, vol. 162, pp. 181-190, 2015.

[11] D. C. Tinker, R. J. Osborne and R. W. Pitz, "Examination of Annular-Electrode Spark Discharges in Flowing Oxygen - An Overview," in AIAA Propulsion and Energy Forum and Exposition, Cincinnati, 2018. 